\title{
Nanotubes from Oxide-Based Misfit Family: The Case of Calcium Cobalt
}

\author{
Oxide \\ Leela S. Panchakarla ${ }^{a, \ddagger}$, Luc Lajaunie $^{b, \ddagger}$, Ashwin Ramasubramaniam ${ }^{c^{*}}$, Raul Arenal $^{b, d^{*}}$ and \\ Reshef Tenne $a^{a^{*}}$ \\ ${ }^{a}$ Department of Materials and Interfaces, Weizmann Institute of Science, 76100 Rehovot, Israel \\ ${ }^{\text {b }}$ Laboratorio de Microscopías Avanzadas, Instituto de Nanociencia de Aragón, Universidad de \\ Zaragoza, 50018 Zaragoza, Spain. \\ ${ }^{\mathrm{C}}$ Department of Mechanical and Industrial Engineering, University of Massachusetts Amherst, \\ Amherst MA 01003, USA \\ ${ }^{\mathrm{d}}$ ARAID Foundation, 50018 Zaragoza, Spain.
}

* Corresponding authors: arenal@unizar.es ; reshef.tenne@weizmann.ac.il

;

$\$$ These authors contributed equally to this work.

\begin{abstract}
Misfit layered compounds (MLCs) have generated significant interest in recent years as potential thermoelectric materials. MLC nanotubes could reveal behavior that is entirely different from the bulk material. Recently, new chemical strategies were exploited for the synthesis of nanotubular forms of chalcogenide-based MLCs, which are promising candidates for thermoelectric materials. However, analogous synthesis of oxide-based MLC nanotubes has not been demonstrated until now. Here, we report a chemical strategy for synthesis of cobalt-oxide-based misfit nanotubes. A combination of high-resolution (scanning) transmission electron microscopy (including image simulations), spatially-resolved electron energy-loss spectroscopy, electron diffraction, and density functional theory (DFT) calculations are used to discover the formation of a phase within these nanotubes that differs significantly from bulk calcium cobaltite MLCs.
\end{abstract}


Furthermore, DFT calculations show that this phase is semiconducting with a band gap in excess of $1 \mathrm{eV}$ unlike bulk calcium cobaltite MLCs, which are known to be metallic. With through systematic experiments, we propose a formation mechanism for these nanotubes that could also apply more generally to realizing other oxide-based MLC nanotubes.

Keywords: Oxide-Based Misfit layered compounds (MLC); Nanotubes; EELS-STEM; DFT

Transition-metal oxides display rich physical phenomena including superconductivity and magnetoresistance, among many others. ${ }^{1}$ Cobalt oxide-based layered structures, in particular, have attracted significant interest for their unconventional transport and magnetic properties ${ }^{2}$ and are being explored for applications ranging from battery cathodes ${ }^{3}$ to thermoelectric devices. ${ }^{4}$ In spite of being metallic, these compounds interestingly display high Seebeck coefficients and thus, high thermoelectric (TE) power. ${ }^{5,6}$ Unconventional two-dimensional superconductivity has also been found in these systems, analogous to layered copper oxides. ${ }^{7}$

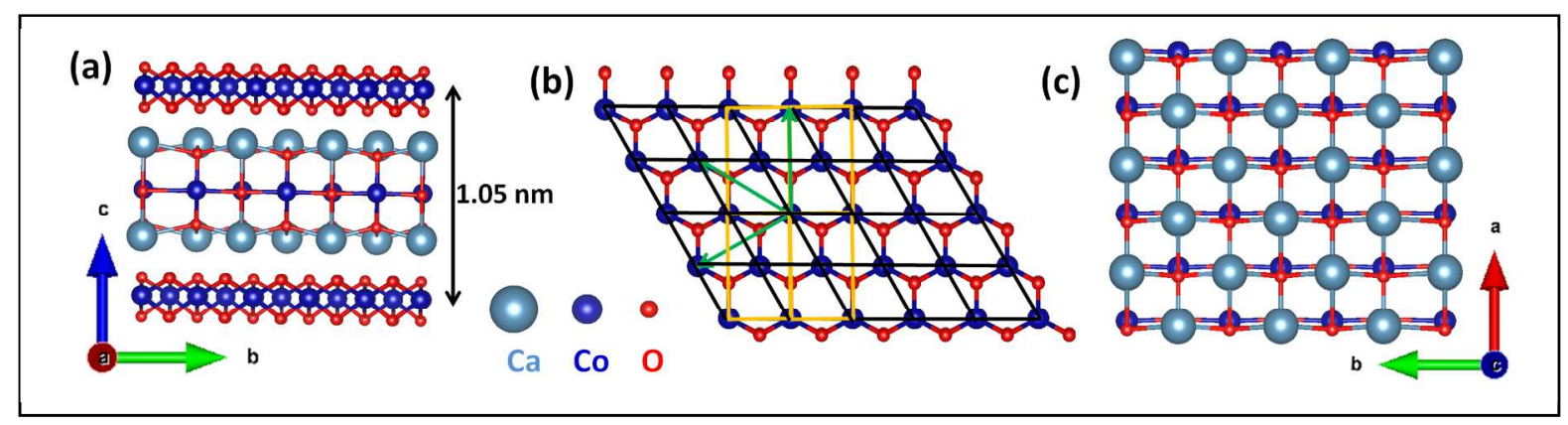

Figure 1 Schematic representation of the crystal structure of layered cobalt oxides (a) $\mathrm{Ca}_{2} \mathrm{CoO}_{3}$ $\mathrm{CoO}_{2},\left(\mathrm{Co}_{3} \mathrm{Ca}_{4} \mathrm{O}_{9}\right)$. (b) A single layer of $\mathrm{CoO}_{2}$ annotated according to pseudo-hexagonal and ortho-hexagonal coordinates shown as black and orange unit cells, respectively. (Equivalent " $b$ " directions are indicated by green arrows.) (c) Schematic model of $\mathrm{Ca}_{2} \mathrm{CoO}_{3}$ single layer. (b) and (c) are viewed along the " $c$ " direction. 
Layered $\mathrm{Ca}_{3} \mathrm{Co}_{4} \mathrm{O}_{9}$ has attracted significant interest as a promising TE material due to its high TE performance, remarkable thermal and chemical stability at elevated temperatures, and its reduced toxicity. ${ }^{6,89} \mathrm{Ca}_{3} \mathrm{Co}_{4} \mathrm{O}_{9}$ has a misfit layered structure (Fig. 1a), consisting of alternate stacking of edge-sharing octahedral $\mathrm{CoO}_{2}$ layers (Fig. 1b) and distorted rocksalt (RS) type $\mathrm{Ca}_{2} \mathrm{CoO}_{3}$ layers (Fig. 1c) along the $c$-axis. ${ }^{6}$ Both subsystems are monoclinic and their lattice parameters coincide along the $a$ and $c$ directions, while differing along the $b$-direction. The lattice mismatch of the two subsystems indicate that $\left[\mathrm{Ca}_{2} \mathrm{CoO}_{3}\right]_{\mathrm{q}}\left[\mathrm{CoO}_{2}\right]$ (denoted hereafter as $\left.\mathrm{Ca}_{2} \mathrm{CoO}_{3}-\mathrm{CoO}_{2}\right)$, is an incommensurately modulated structure with $\mathrm{q}=b_{2} / b_{1}$ denoting the misfit parameter, $b_{1}$ and $b_{2}$, being the lattice parameters of $\mathrm{Ca}_{2} \mathrm{CoO}_{3}$ and $\mathrm{CoO}_{2}$, respectively, in the $b$ direction). Previous studies confirmed the misfit parameter $(\mathrm{q})$ to be around $0.62 .{ }^{6,10}$ It should be nevertheless noted that misfit compounds are not fully commensurate and are therefore not strictly stoichiometric compounds. Approximate composition of misfit compounds varies slightly with the reaction conditions. The ratio between the area occupied by the unit cell of the two components in the bulk, i.e. $\mathrm{Ca}_{2} \mathrm{CoO}_{3}$ (1) and $\mathrm{CoO}_{2}$ (2) is $(\mathrm{q}=0.62)$ is translated into the approximate composition $\mathrm{Ca}_{3} \mathrm{Co}_{4} \mathrm{O}_{9}$.

Generally, the TE performance of materials improves with decreasing dimensionality. ${ }^{11}$ The inherent layered structure and interlayer misfit make misfit layered compounds (MLCs) ideal candidates for synthesis of low-dimensional structures, including nanotubular forms, for TE applications. Tubular structures of chalcogenide-based MLCs have been synthesized as nanotubes and studied recently. ${ }^{12-14}$ However, one-dimensional analogues of oxide misfit compounds have not been known hitherto.

In this work, we report the synthesis of misfit oxide nanotubes from calcium cobalt oxide. Through a combination of high-resolution (scanning) transmission microscopy, electron 
diffraction, and density functional theory (DFT) calculations we uncover a calcium-oxidedeficient phase of calcium cobalt oxide consisting of a $\mathrm{CaCoO}_{2}$ layer sandwiched between hexagonal $\mathrm{CoO}_{2}$ layers. DFT calculations predict an antiferromagnetic coupling between the $\mathrm{CaCoO}_{2}$ and $\mathrm{CoO}_{2}$ layers and, importantly, reveal the presence of a semiconducting gap $(\sim 1 \mathrm{eV})$ unlike bulk calcium oxide MLCs, which are metallic. Through detailed studies, we postulate a formation mechanism for these nanotubes that could potentially be exploited for similar syntheses of other oxide MLC nanotubes. Overall, these calcium cobalt oxide nanotubes reported here hold promise for TE applications and could also provide a platform for fundamental studies of low-dimensional oxides.

\section{RESULTS AND DISCUSSION}

Fig. 2a shows the products of the hydrothermal synthesis of $\mathrm{Ca}_{3} \mathrm{Co}_{4} \mathrm{O}_{9}$ in basic conditions. Several tubular nanostructures (marked by red arrows in Fig. 2a) are observed with typical lengths of several hundred nanometers and external diameters close to $50 \mathrm{~nm}$. From SEM images and low-magnification TEM micrographs (and Fig. S2 and S3 in the SI), the yield of the nanotubes is estimated to be around 20-30 \%. Fig. 2b displays a HR-STEM HAADF micrograph of an edge of one of these nanotubes from which we observe a high degree of crystallinity. In particular, going from the center towards the surface, an alternation of bright atomic layers with a periodicity of $0.86 \mathrm{~nm}$ is clearly highlighted. This is the most commonly observed periodicity for these nanotubes (see Fig. S4 in SI) and is significantly smaller than the $1.05 \mathrm{~nm}$ period expected from the $\mathrm{Ca}_{3} \mathrm{Co}_{4} \mathrm{O}_{9}$ starting material (Fig. 1a). This fact suggests that a layer from the initial bulk structure is now missing. Fig. 2c shows a HR-STEM HAADF micrograph of the 
same nanotube obtained at higher magnification than the one of Fig. 2 b. The corresponding intensity profile, obtained following the marked line of Fig. 2c, is depicted in Fig. 2d. Sandwiched between the bright layers, spaced by $0.86 \mathrm{~nm}$ (labeled $X$ on Fig. 2c and 2d), two other atomic layers, with weaker intensities (highlighted by purple arrows on Fig. 2c and labeled $y$ and $z$ on in Fig. 2d, respectively) can be distinguished. The three features $(X, y$ and $z)$ display different intensities, indicating a stacking of three different layers. All of these results suggest that the nanotubes consist of a different phase than the bulk starting material.

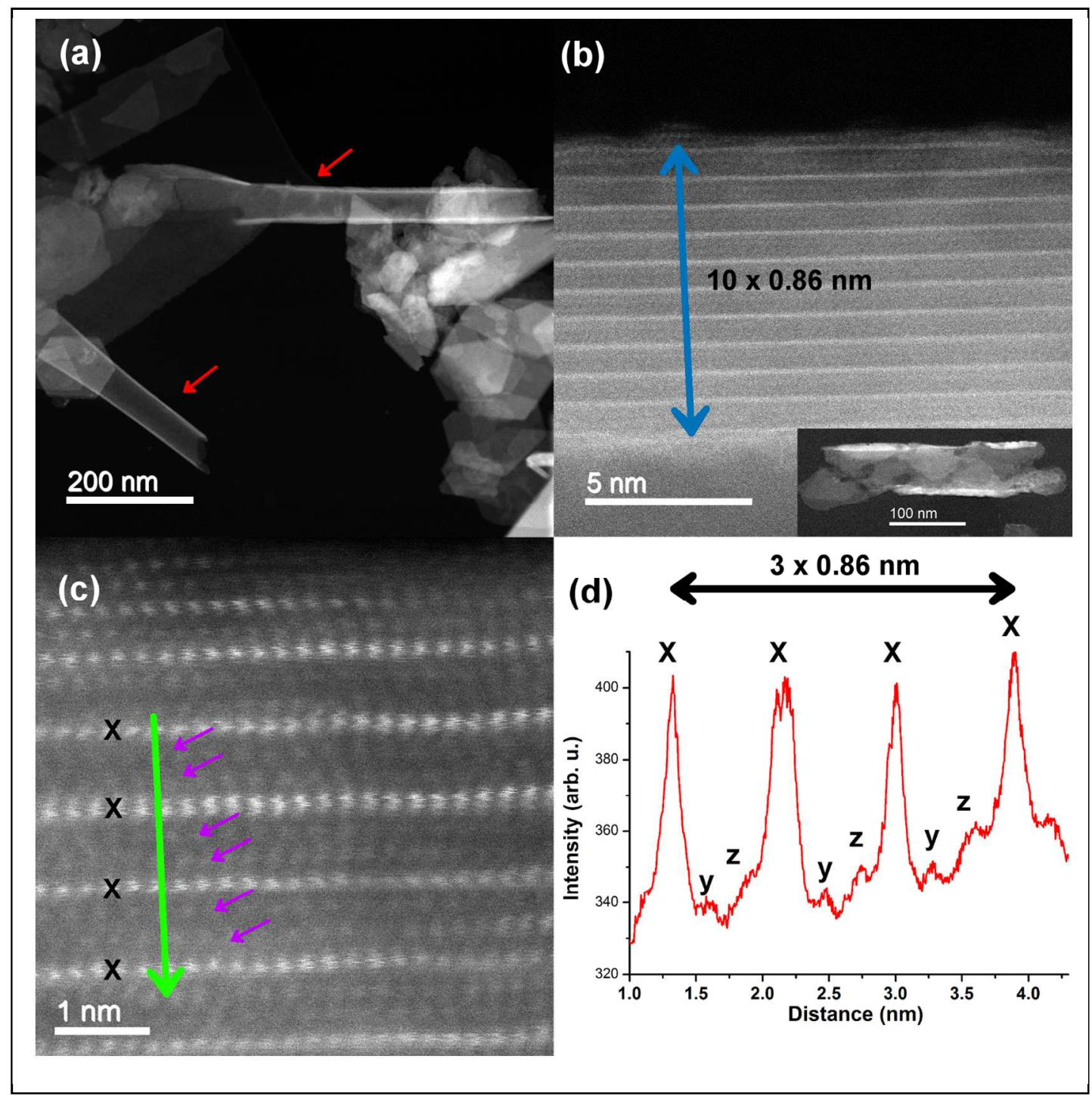


Figure 2.(a) Low-Magnification STEM HAADF micrograph of the products coming from the hydrothermal synthesis of $\mathrm{Ca}_{3} \mathrm{Co}_{4} \mathrm{O}_{9}$ in basic conditions. The red arrows draw attention to the tubular nanostructures. (b) HR-STEM HAADF micrograph of the upper edge of the nanotube shown in the inset, highlighting the $0.86 \mathrm{~nm}$-periodicity of the bright layers. (c) HR-STEM HAADF image of the same area as (b) but with a higher magnification. The purple arrows highlight the atomic layers sandwiched between the bright layers. The green arrow indicates the area from which the intensity profile in (d) was obtained.

To achieve further insight into the chemical nature of this missing layer, EELS and SR-EELS elemental quantification were performed by using the $\mathrm{Ca}-\mathrm{L}_{2,3}, \mathrm{O}-\mathrm{K}$ and $\mathrm{Co}-\mathrm{L}_{2,3}$ edges situated around 349, 530 and $775 \mathrm{eV}$, respectively (Fig. 3a). ${ }^{15}$ Quantification performed at the center of the nanotube yields atomic concentrations of 13, 37, and 50 at.\% for $\mathrm{Ca}$, $\mathrm{Co}$ and $\mathrm{O}$ respectively. This indicates a deficiency in calcium and oxygen when compared to the bulk starting material (expected concentrations of 19, 25 and 56 at.\% for $\mathrm{Ca}$, Co and $\mathrm{O}$ respectively). However, proper quantification by EELS analysis is not straightforward, as accurate quantification requires the use of reference materials. ${ }^{16}$ Another complementary approach is to combine chemical and spatial variations to determine the chemical structure at the local scale. This approach has been successfully used on other misfit nanotubes. ${ }^{17}$ Fig. 3c shows the superposition of the Ca/Co chemical map (obtained from SR-EELS analyses) and the ADF micrograph obtained at the same time on the edge of one nanotube (Fig. 3b). The bright layers, corresponding to the $X$ features in the intensity profile (Fig. 3c), perfectly match the areas with the lowest $\mathrm{Ca} / \mathrm{Co}$ ratio and correspond to one of the two cobalt sub-systems $\left(\mathrm{CoO}_{2}\right.$ or $\left.\mathrm{CoO}\right)$. Just below these Co-rich layers, other layers, showing the highest $\mathrm{Ca} / \mathrm{Co}$ ratios, can be clearly observed. These layers correspond to the $y$ features in the intensity profile and are therefore related to the $\mathrm{Ca}$ sub-system $(\mathrm{CaO})$. The $\mathrm{Ca} / \mathrm{Co}$ ratio of the other layers (highlighted by red arrows on Fig. $\mathbf{3 c}$ and $\mathbf{3 d}$ and corresponding to the $z$ features in the intensity profile) shows an intermediate behavior between the Co- and Ca- 


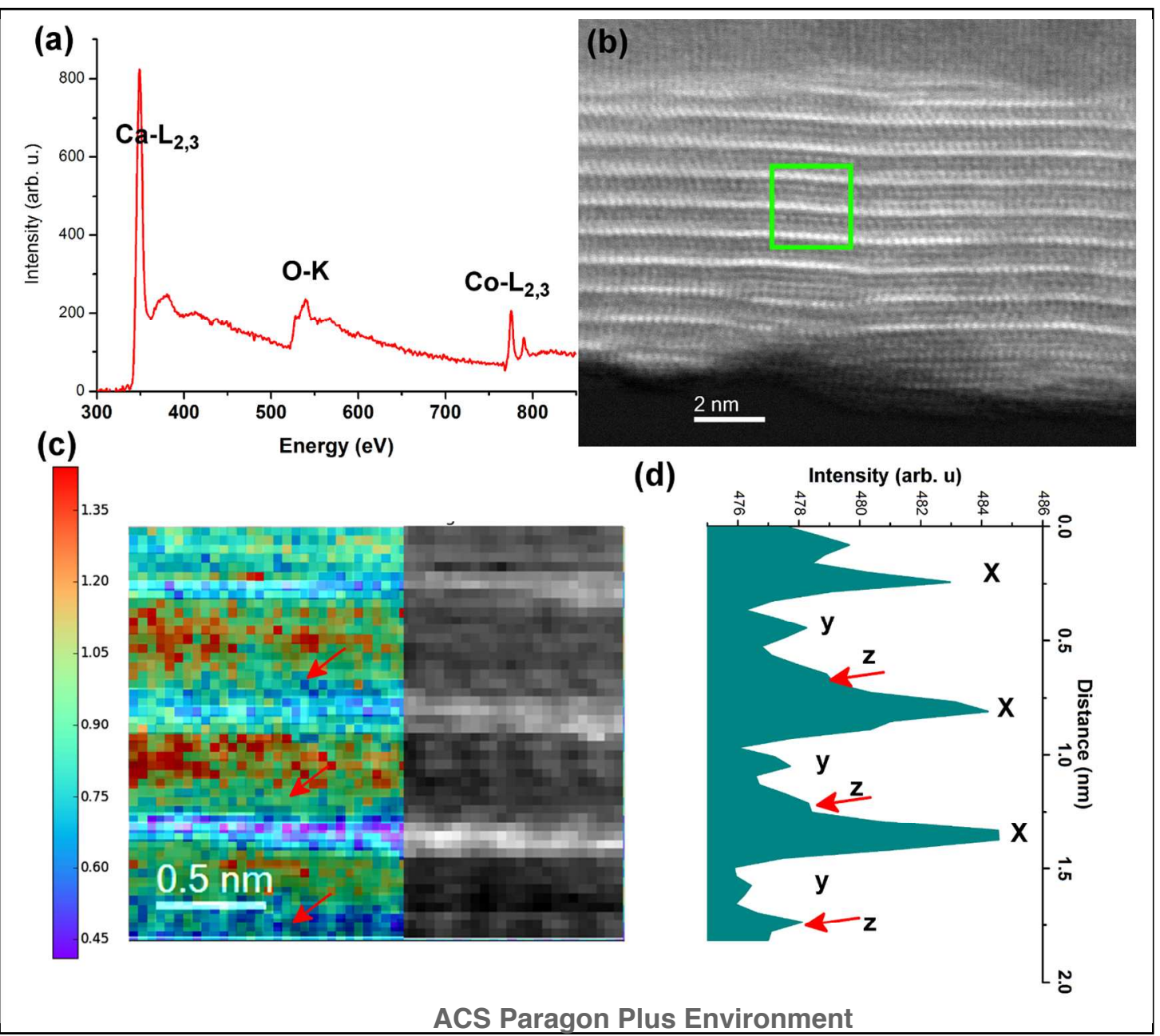

richest layers. This behavior could not be ascribed to the presence of another $\mathrm{CaO}$ layer but is more likely related to the remaining cobalt sub-system $\left(\mathrm{CoO}_{2}\right.$ or $\left.\mathrm{CoO}\right)$. In addition, the $X$ and $z$ layers also coincide with an increase of the $\mathrm{Co} / \mathrm{O}$ ratio, while the $y$ layers coincide with an increase of the $\mathrm{Ca} / \mathrm{O}$ ratio (Fig. $\mathbf{S} 4$ in the $\mathbf{S I}$ ). All these results have been checked and observed in other areas/NTs, confirming the assignment of the $X$ and $z$ layers to Co sub-systems and the $y$ layers to the $\mathrm{Ca}$ sub-system. Thus, from the SR-EELS analyses, it can be concluded that $\mathrm{CaO}$ is the missing layer when comparing with the reference bulk material. The nanostructured tubular form of $\mathrm{Ca}_{3} \mathrm{Co}_{4} \mathrm{O}_{9}$ (equivalent to the misfit structure $\mathrm{Ca}_{2} \mathrm{CoO}_{3}-\mathrm{CoO}_{2}$ ) thus leads to the formation of a phase $\mathrm{CaCoO}_{2}-\mathrm{CoO}_{2}(17,33$, and 50 at.\% for $\mathrm{Ca}$, $\mathrm{Co}$ and $\mathrm{O}$ respectively). This finding is also in excellent agreement with the elemental quantification performed by EELS (atomic concentrations of 13,37 , and 50 at.\% for $\mathrm{Ca}$, Co and $\mathrm{O}$ respectively).

(c) 
Figure 3. (a) Typical EELS spectra acquired at the center of a nanotube. (b) Lower-edge of the same nanotube shown in Fig. 2. The green square highlights the area used for the SR-EELS analysis. (c) Superposition of the $\mathrm{Ca} / \mathrm{Co}$ ratio map obtained from SR-EELS (left) and the HRSTEM ADF micrograph (right) acquired at the same time. (d) Corresponding intensity profile obtained from the ADF image.

To understand the variations in the crystal structure, we collected and analyzed the selected area electron diffraction (SAED) on a single nanotube. Fig. 4a shows a typical HRTEM image of a $\mathrm{CaCoO}_{2}-\mathrm{CoO}_{2}$ nanotube and the corresponding SAED pattern is shown in Fig. 4b. A low magnification TEM image of the entire nanotube is shown as an inset in Fig. 4a. A periodicity of $0.86 \mathrm{~nm}$ is once again observed (see the intensity profile at the bottom of Fig. 4a), confirming the superstructure of alternating $\mathrm{CaCoO}_{2}$ and $\mathrm{CoO}_{2}$ layers. SAED also confirmed the superstructure periodicity (Fig. 4b). The tube axis and basal reflections are indicated in Fig. 4b using green and white arrows, respectively. Spots corresponding to the same interplanar spacing are marked by segmented circles/ellipses. The measured interplanar values with corresponding Miller indices are indicated. Blue and yellow segmented circles/ellipses correspond to the $\mathrm{CaCoO}_{2}$ and $\mathrm{CoO}_{2}$ sub-systems, respectively, while the red segmented ellipses corresponds to the combined $d$-spacing. There are four pairs of $\{110\}$ and $\{220\}$ reflections of $\mathrm{CaCoO}_{2}$ on a circle, equal azimuthally distributed with $d$-spacing of 3.62 and $1.83 \AA$, respectively. The multiplicity factor of these planes is four, indicating that there is only one type of $\mathrm{CaCoO}_{2}$ sheet present in the tube. This single orientation of the RS layer is quite uncommon in chalcogenide MLC nanotubes. ${ }^{12}$ The four sets of spots are splintered by similar angles $\left(20^{\circ}\right)$; such a splitting arises from the chiral folding of the layers with a chiral angle of $10^{\circ}$, which is equal to half of the 


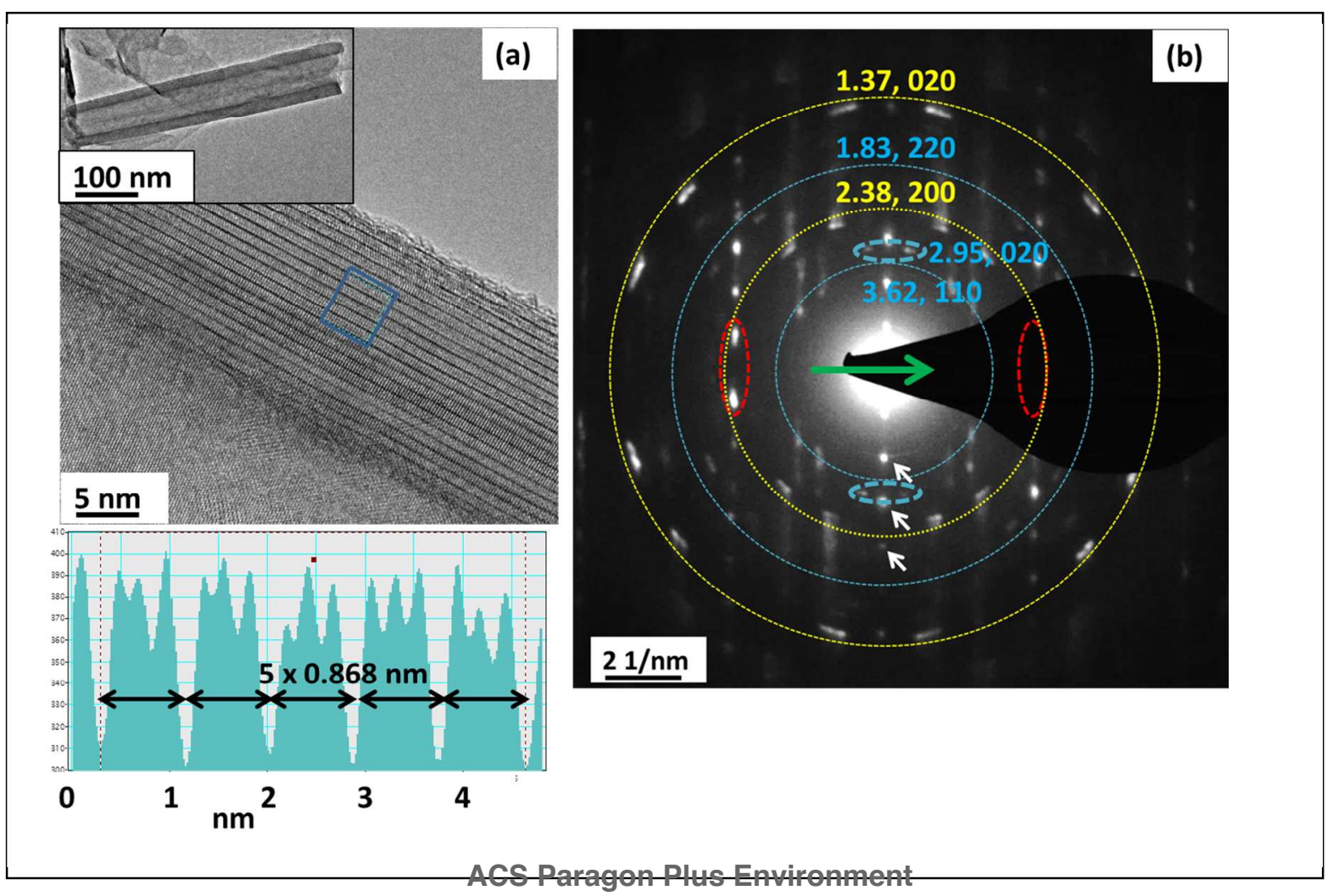

azimuthal splitting of the spots. In misfit compounds, it is generally observed that the MX layers tend to adapt to the $\mathrm{TX}_{2}$ layers (also known in the literature as "structural modulation"). Thus, it is appropriate to define the pseudo-hexagonal $\mathrm{CoO}_{2}$ with ortho-hexagonal unit cell using lattice parameter of $a, b=\sqrt{ } 3 a$ and $c$ (Fig. 1b). There are six sets of $\{200\}$ (corresponding to the 11.0 hexagonal notation, i.e. "armchair") and $\{020\}$ (corresponding to the 10.0 in the hexagonal notation, i.e. "zigzag") reflections of $\mathrm{CoO}_{2}$ equally distributed azimuthally on the circle with $d$ spacing of 2.38 and $1.37 \AA$, respectively. The chiral angles of the layers are $10.5^{\circ}$ in this case. The $a$-directions of $\mathrm{CaCoO}_{2}$ and $\mathrm{CoO}_{2}$ are parallel, and the lattice parameters are commensurate in this direction. Thus, two sets of spots out of six sets of observed $\{200\}$ planes with a $d$ spacing of $2.38 \AA$ are common to both the $\mathrm{CaCoO}_{2}$ and the $\mathrm{CoO}_{2}$ layers coinciding also with the tube axis. These spots are marked by red dashed ellipses in Fig. $\mathbf{4 b}$. There are two pairs of $\{020\}$ reflections of $\mathrm{CaCoO}_{2}$ with $d$-spacing of $2.95 \AA$, which is $30 \%$ higher than the bulk counterpart of $\mathrm{Ca}_{2} \mathrm{CoO}_{3}(2.278)^{6}$ in $\mathrm{Ca}_{3} \mathrm{Co}_{4} \mathrm{O}_{9}$. 
Figure4. (a) HRTEM image of a $\mathrm{CaCoO}_{2}-\mathrm{CoO}_{2}$ nanotube, shown alongside a line profile (bottom) obtained from the region indicated by the blue rectangle. A low magnification TEM image of the same tube is shown as an inset. (b) SAED pattern taken from the tube shown in (a). The tube axis and basal ( $c$-axis) reflections are marked with green and white arrows, respectively. Spots corresponding to the same interplanar spacings are marked by segmented circles/ellipses and measured interplanar values with corresponding Miller indices are indicated. The blue and yellow segmented circles/ellipses indicate reflections from the $\mathrm{CaCoO}_{2}$ and $\mathrm{CoO}_{2}$ sub-systems, respectively, while the red segmented ellipses indicates spots originating from both sub-systems.

DFT calculations were performed to understand the obtained crystal structure and its properties. As in the case of the well-known $\mathrm{Ca}_{3} \mathrm{Co}_{4} \mathrm{O}_{9}(\mathrm{CCO})$ structure, the $\mathrm{CaCoO}_{2}-\mathrm{CoO}_{2}$ structure also consists of two subsystems that are experimentally measured to be commensurate along the $a$ direction $(4.76 \AA)$ but incommensurate along the $b$ direction $\left(\mathrm{CaCoO}_{2}-5.90 \AA\right.$, $\mathrm{CoO}_{2}-2.74 \AA$ ). To obtain an appropriate simulation cell, we created a structure with composition $\left(\mathrm{CaCoO}_{2}\right)_{\mathrm{b} 1}-\left(\mathrm{CoO}_{2}\right)_{\mathrm{b} 2}$ where $b_{1}$ and $b_{2}$ are the periodicities of each subsystems along the $b$ axis. We chose $b_{1}=1$ and $b_{2}=2$, which results in a tractable computational supercell with a mismatch strain of $\sim 7 \%$ between the two subsystems; this is the same level of strain in the commonly studied 3/2 rational approximant of $\mathrm{CCO} .{ }^{18}$ The initial structure of the $\mathrm{CaCoO}_{2}$ layer was produced simply by eliminating one $\mathrm{CaO}$ layer from the $\mathrm{Ca}_{2} \mathrm{CoO}_{3} \mathrm{RS}$ structure. We assume here that the bulk structural model represents a reasonable surrogate for the actual nanotubes. This approximation is expected to be valid as long as (a) the nanotube diameters are sufficiently large for curvature effects to be negligible, and (b) the nanotubes consist of a sufficient number 
of layers to approach a bulk-like limit. Both of these conditions are generally met for the synthesized structures in the present study.

To confirm the $\mathrm{CaCoO}_{2}-\mathrm{CoO}_{2}$ structure, derived from DFT structural optimization, we have performed a comparison between the experimental and simulated HR-STEM HAADF images, using the DFT-relaxed structure as input for the simulation. Fig.5 shows the relaxed atomic structure of $\mathrm{CaCoO}_{2}-\mathrm{CoO}_{2}$ obtained from DFT calculations superposed over the simulated HRSTEM HAADF micrograph; also displayed are the experimental image and the comparison of the experimental and simulated intensity line profiles. As seen, we find excellent agreement between the simulated and experimental micrographs. Not only are the distances between the $X$, $y$ and $z$ layers well reproduced but so are their respective intensities. In addition, Fig. 5 clearly shows that the $X, y$ and $z$ layers are related to $\mathrm{CoO}_{2}, \mathrm{CaO}$ and $\mathrm{CoO}$ layers, respectively. The excellent consistency between high-resolution microscopy, spatially-resolved EELS spectroscopy, and DFT calculations support and confirm our proposed structure for the calcium cobaltite phase. The consistency with experiments also justifies the choice of simulation cell for more detailed DFT analyses presented next. 
(a)

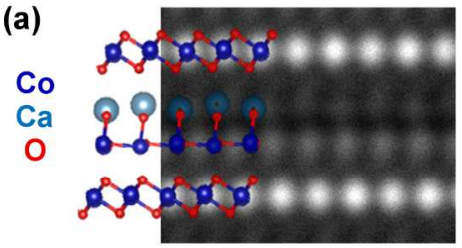

(b)
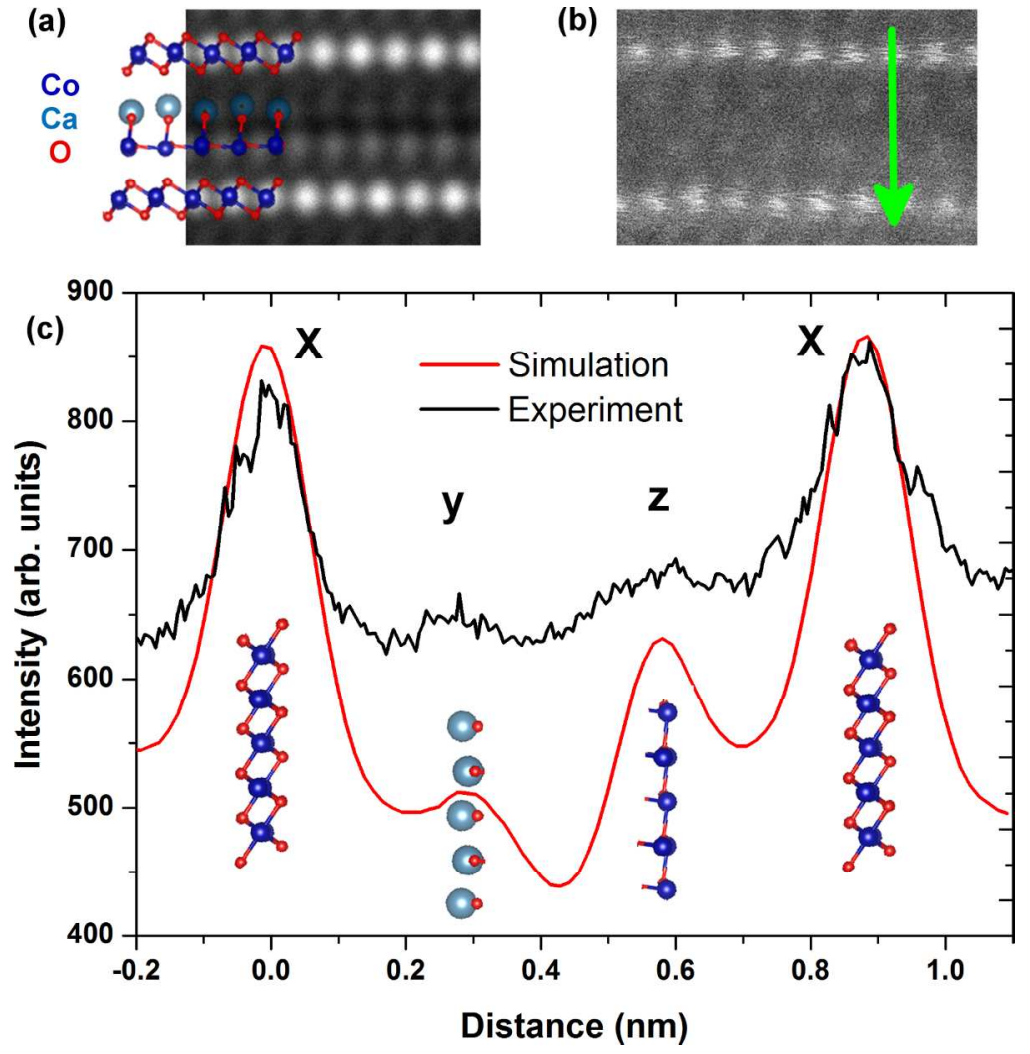

Figure 5. (a) Simulated HR-STEM HAADF micrograph superposed with the relaxed atomic structure of $\mathrm{CaCoO}_{2}-\mathrm{CoO}_{2}$ used as input. (b) Experimental STEM HAADF image. The green arrow highlights the direction used to determine the experimental and simulated intensity line profiles displayed in $(\mathrm{c})$. 


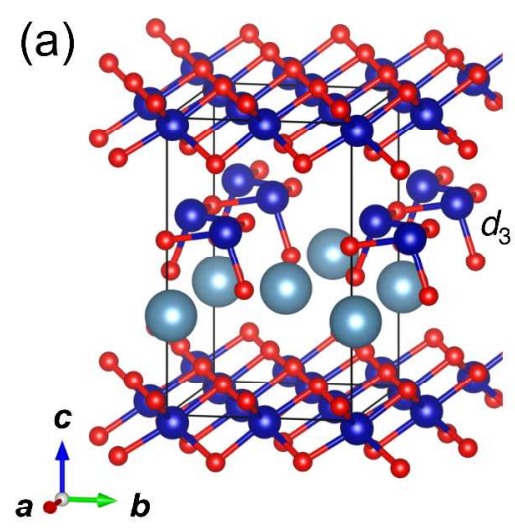

(c)

(b)
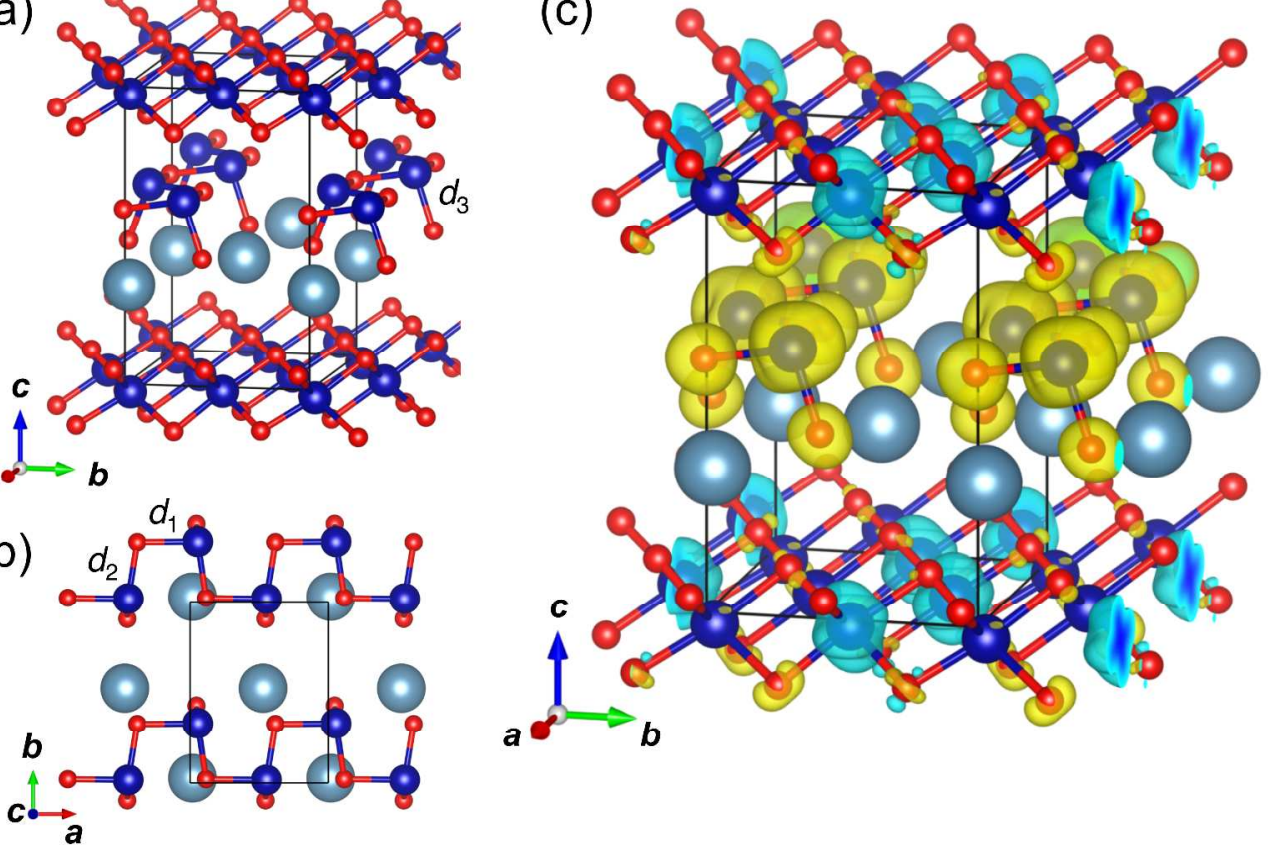

Figure 6. (a) Relaxed structure of $\left(\mathrm{CaCoO}_{2}\right)-\left(\mathrm{CoO}_{2}\right)_{2}$ supercell. (b) View of relaxed $\mathrm{CaCoO}_{2}$ layer along the $c$-axis. $\mathrm{Ca}, \mathrm{Co}$, and $\mathrm{O}$ atoms are indicated by (large) teal, dark blue, and red spheres, respectively. The $\mathrm{Co}$ atoms in the $\mathrm{CaCoO}_{2}$ layer are threefold-coordinated: staggered Co-O dimers $\left(d_{1}=1.95 \AA\right)$ are aligned along the $a$-axis with bridging Co-O bonds between parallel rows $\left(d_{2}=1.85 \AA\right)$; the third Co-O bond is out of plane $\left(d_{3}=1.83 \AA\right.$ ). (c) Isosurfaces of spin density within the relaxed $\left(\mathrm{CaCoO}_{2}\right)-\left(\mathrm{CoO}_{2}\right)_{2}$ supercell. Yellow/cyan surfaces correspond to densities of $\pm 0.067 \mathrm{e} / \AA^{3}$. As seen, the majority spin density resides almost entirely on the $\mathrm{CaCoO}_{2}$ layer while the minority spin density is localized on the $\mathrm{CoO}_{2}$ layer.

As noted previously, the $\mathrm{CaCoO}_{2}$ layer can be obtained by removing one plane of $\mathrm{CaO}$ atoms from the conventional $\mathrm{RS} \mathrm{Ca}_{2} \mathrm{CoO}_{3}$ structure. Starting from this initial guess, we performed structural relaxation calculations (with $\mathrm{PBE}+\mathrm{U}$ ) to obtain the optimized supercells displayed in Figs.6(a) and 6(b). Atomic positions and lattice vectors for this relaxed structure are provided in 
the SI (Table S1). It is worth noting that the computed $c$-axis lattice parameter value of $0.898 \mathrm{~nm}$ (Table S1) agrees very well with the measured value of $0.86 \mathrm{~nm}$, which supports the validity of the proposed model for the $\mathrm{Ca}_{2} \mathrm{CoO}_{3}$ layer with one $\mathrm{CaO}$ layer missing relative to the original $\mathrm{RS}$ structure. The agreement might be further improved with larger supercell approximants that reduce the lattice mismatch strain. From Fig. 6, it is readily apparent that the $\mathrm{CaCoO}_{2}$ layer develops a cluster-like structural pattern when viewed along the $a$-axis, analogous to the observations by Rébola et $a l .{ }^{18}$ for the RS substructure of CCO. Each Co atom within the $\mathrm{CaCoO}_{2}$ layer is now threefold coordinated with the $\mathrm{O}$ atoms: staggered Co-O dimers (1.95 $\left.\mathrm{A}\right)$ are arranged in parallel rows along the crystal $a$-axis with bridging Co-O bonds $(1.85 \AA$ ) between the rows; the remaining Co-O bond is out of plane (1.83 $\AA$ ), the corresponding $\mathrm{O}$ atom also being coordinated with a $\mathrm{Ca}$ atom. The overall structure of the $\mathrm{CaCoO}_{2}$ layer is thus distinctly anisotropic and may be viewed as consisting of quasi-1D, puckered chains along the $a$-axis. The trigonal monopyramidal coordination of the Co atom is also relatively unusual unlike the more common trigonal bipyramidal ${ }^{19}$ or distorted octahedral ${ }^{18}$ coordination in $\mathrm{CCO}$. The $\mathrm{CoO}_{2}$ layer is essentially unaffected by the removal of the $\mathrm{CaO}$ plane and retains the same structure as in $\mathrm{CCO}$.

Figure $\mathbf{S 5}$ in the SI displays the electronic density of states (DOS) for the $\left(\mathrm{CaCoO}_{2}\right)-\left(\mathrm{CoO}_{2}\right)_{2}$ structure. Unlike $\mathrm{CCO}$ which is metallic, we find that $\left(\mathrm{CaCoO}_{2}\right)-\left(\mathrm{CoO}_{2}\right)_{2}$ is essentially semiconducting: the Fermi level clearly lies within the band gap of the minority spin channel and, up to numerical error $(\sim 20 \mathrm{meV})$ it also lies at the very edge of the valence band for the majority spin channel. The calculated $\mathrm{PBE}+\mathrm{U}$ band gap for the majority spin channel is $\sim 1.19 \mathrm{eV}$ while that for the minority spin channel is $\sim 1.30 \mathrm{eV}$. We also observe from the DOS that there is an excess of majority spin carriers at the valence band edge, which is consistent with the overall calculated magnetic moment of $6 \mu_{\mathrm{B}}$ for the entire supercell, and that the dominant contribution to 
the net magnetic moment arises from the $\mathrm{CaCoO}_{2}$ layer. The spin density distribution is depicted in Fig. 6(c) from which we note positive magnetic moments on all atoms in the $\mathrm{CaCoO}_{2}$ layer and localized negative magnetic moments on $\mathrm{Co}$ atoms in the $\mathrm{CoO}_{2}$ layer. Thus, the $\mathrm{CaCoO}_{2}$ and $\mathrm{CoO}_{2}$ layers are antiferromagnetically coupled, which is similar to that in $\mathrm{CCO},{ }^{18,19}$ leading overall to a ferrimagnetic ground state. It should be noted that magnetic measurements of the product were carried out, but they could not give a conclusive result since the yield of the nanotubes was not high enough and the chemical composition and the magnetic response of the side products, like $\mathrm{CoO}_{2}$ platelets, is entirely different from that of the tubes.

Formally, excluding considerations of charge transfer between the $\mathrm{CaCoO}_{2}$ and $\mathrm{CoO}_{2}$ layers for the moment, one would expect the $\mathrm{Co}$ atoms to be in a $\mathrm{Co}^{2+}$ oxidation state in the $\mathrm{CaCoO}_{2}$ layer with either a high-spin $\left(3 \mu_{\mathrm{B}}\right)$ or a low-spin $\left(1 \mu_{\mathrm{B}}\right)$ state. By integrating the spin density over the atomic (Bader) volumes, we find that each $\mathrm{Co}$ atom in the $\mathrm{CaCoO}_{2}$ layer indeed carries a magnetic moment of $3.03 \mu_{\mathrm{B}}$ (see Table S1 in SI). Figure S5(b) in SI displays the density of states projected over the $d$-orbitals of these Co atoms from which we observe that the dominant contributions to the Co spin arise from unpaired electrons in the $e_{g}$ orbitals with smaller contributions from the $t_{2 \mathrm{~g}}$ (primarily $d_{\mathrm{xy}}$ and $d_{\mathrm{yz}}$ ) orbitals. Similarly, excluding charge transfer considerations, one would expect a $\mathrm{Co}^{4+}$ oxidation state for the $\mathrm{Co}$ atoms in the $\mathrm{CoO}_{2}$ layer leading to a magnetic moment of $1 \mu_{\mathrm{B}}$. Integrating the spin density over Bader volumes however shows that two of the Co atoms possess a magnetic moment of $-1.08 \mu_{\mathrm{B}}$ whereas the other two Co atoms possess magnetic moments of $0.02 \mu_{\mathrm{B}}$ (see Table S1 in SI); the two differently spinpolarized Co atoms are readily distinguished from the spin-density contours in Fig. $6(\mathbf{c})$. This is then indicative of the $\mathrm{Co}$ atoms in the $\mathrm{CoO}_{2}$ layer being in a low-spin, mixed valence state of $\mathrm{Co}^{3+}$ and $\mathrm{Co}^{4+}$ in a 1:1 ratio analogous to the results of Rébola et al. ${ }^{18}$ for CCO. 
The formation of the mixed valence $\mathrm{Co}^{3+}$ and $\mathrm{Co}^{4+}$ oxidationstates within the $\mathrm{CoO}_{2}$ layer depends sensitively upon charge transfer from the $\mathrm{CaCoO}_{2}$ layer, which can be estimated using a Bader analysis. By integrating the atomic charges over the $\mathrm{CaCoO}_{2}$ and $\mathrm{CoO}_{2}$ layers (see Table S1 in SI), we find that there is net transfer of $1.57 \mathrm{e}^{-}$from the $\mathrm{CaCoO}_{2}$ layer to the $\mathrm{CoO}_{2}$ layer. A more detailed analysis of atomic charge distribution reveals that each $\mathrm{Ca}$ atom donates, on average, $1.59 \mathrm{e}^{-}\left(3.18 \mathrm{e}^{-}\right.$total $)$; of these, $1.61 \mathrm{e}^{-}$are redistributed within the $\mathrm{CaCoO}_{2}$ layer while the remaining $1.57 \mathrm{e}^{-}$are transferred to the $\mathrm{CoO}_{2}$ layer $\left(0.39 \mathrm{e}^{-}\right.$per formula unit). It is this excess charge transferred to the $\mathrm{CoO}_{2}$ layer, which then leads to mixed valence $\mathrm{Co}^{3+}$ and $\mathrm{Co}^{4+}$ state of Co atoms. A similar reckoning for the $3 / 2$ rational approximant ${ }^{18}$ for $\mathrm{CCO}$, shows that the $\mathrm{CoO}_{2}$ layer acquires a much higher charge of $1.33 \mathrm{e}^{-}$per formula unit due to the presence of additional donor $\mathrm{Ca}$ atoms within the $\mathrm{RS} \mathrm{Ca}_{2} \mathrm{CoO}_{3}$ layer relative to the $\mathrm{CaCoO}_{2}$ layer (see Table $\mathbf{S 2}$ in $\mathbf{S I}$ ). The ratio of $\mathrm{Co}^{3+}$ to $\mathrm{Co}^{4+}$ in $\mathrm{CCO}$ is proportionately higher at 5:1 in comparison to $1: 1$ for $\left(\mathrm{CaCoO}_{2}\right)-\left(\mathrm{CoO}_{2}\right)_{2}$. Thus, we conclude that the $\mathrm{Ca}$ atoms act primarily as a charge reservoir and it should be possible to control the overall metallic versus semiconducting nature of the misfit compound via the concentration of the Ca atoms and/or other donor species.

The formation of misfit chalcogenide nanotubes belonging to the $\mathrm{MS}^{-} \mathrm{TS}_{2}$ phases, like PbS$\mathrm{TaS}_{2}$ or LaS-CrS 2 , was attributed to two independent stimuli. ${ }^{12-14}$ The mismatch between the distorted rocksalt MS and the hexagonal $\mathrm{TS}_{2}$ layers is partially relieved by folding and scrolling. Additionally, the dangling bonds of the atoms on the prismatic $(h k 0)$ planes of the nanoparticles can be passivated by seaming the folded layers. The combination of these two independent driving forces provides sufficient energy at high temperatures to overcome the elastic energy cost of bending leading to the formation of MLC nanotubes. However, under the present hydrothermal conditions, where the temperature is limited to $200{ }^{\circ} \mathrm{C}$, the thermal energy is 
unlikely to be sufficient to overcome the energy barrier of a complete folding and would thus inhibit the formation of such nanotubes. Fortunately though, the chemical reaction provides another stimulus for the folding of the layers.

To understand the formation mechanism of our oxide MLC nanotubes, the duration of the hydrothermal synthesis was varied and the product was systematically characterized by TEM and SAED. Fig. S6 in the supporting information displays TEM images of the products obtained in the hydrothermal reaction at $200{ }^{\circ} \mathrm{C}$ for $4 \mathrm{~h}$. Partial rolling of the nanosheets can be clearly seen in the TEM images. Since TEM provides reliable evidence that the oxide MLC nanotubes were made from thin sheets, it becomes vital to comprehend the driving force that bend the nanosheets. The following formation mechanism is proposed for the nanotubes (Fig.7). When the $\mathrm{NaOH}$ solution is mixed with the cobalt oxide-based misfit compounds, $\mathrm{Na}^{+}$and $\mathrm{OH}^{-}$ions intercalate between the layers and expand the sheets. Partial dissolution of the $\mathrm{CaO}$ takes places during this process. Since, the resultant expanded sheet lacks inversion symmetry, an asymmetric environment results around the layer that generates the intrinsic tension. The relaxation of strain energy provides the driving force to curve the layered structure. The realization of the asymmetry along the $c$-axis in layered compounds, like chrysotile, led Pauling to propose ${ }^{20}$ that they would spontaneously fold and form nanotubes, a prediction that was vindicated only two decades later. ${ }^{21}$ A similar mechanism was proposed for the formation of $\mathrm{H}_{2} \mathrm{Ti}_{3} \mathrm{O}_{7}$ nanotubes, ${ }^{22}$ where Peng and his co-workers showed that the alkaline environment in the hydrothermal reaction leads to $\mathrm{H}$ abstraction from the top layer of the $\mathrm{H}_{2} \mathrm{Ti}_{3} \mathrm{O}_{7}$ crystallite surface. This asymmetry facilitates the exfoliation and folding of this top layer, which becomes a nanotube upon seaming of the edges. Somewhat similar conditions hold also for the synthesis of hexaniobate $\left(\mathrm{K}_{4} \mathrm{Nb}_{6} \mathrm{O}_{17}\right)$ nanotubes. ${ }^{23}$ This chemically induced asymmetry comes on top of the 
misfit stress, which is inherent to the MLC structure, further helping to curve the $\mathrm{CaCoO}_{2}-\mathrm{CoO}_{2}$ layer into a nanotubular structure.

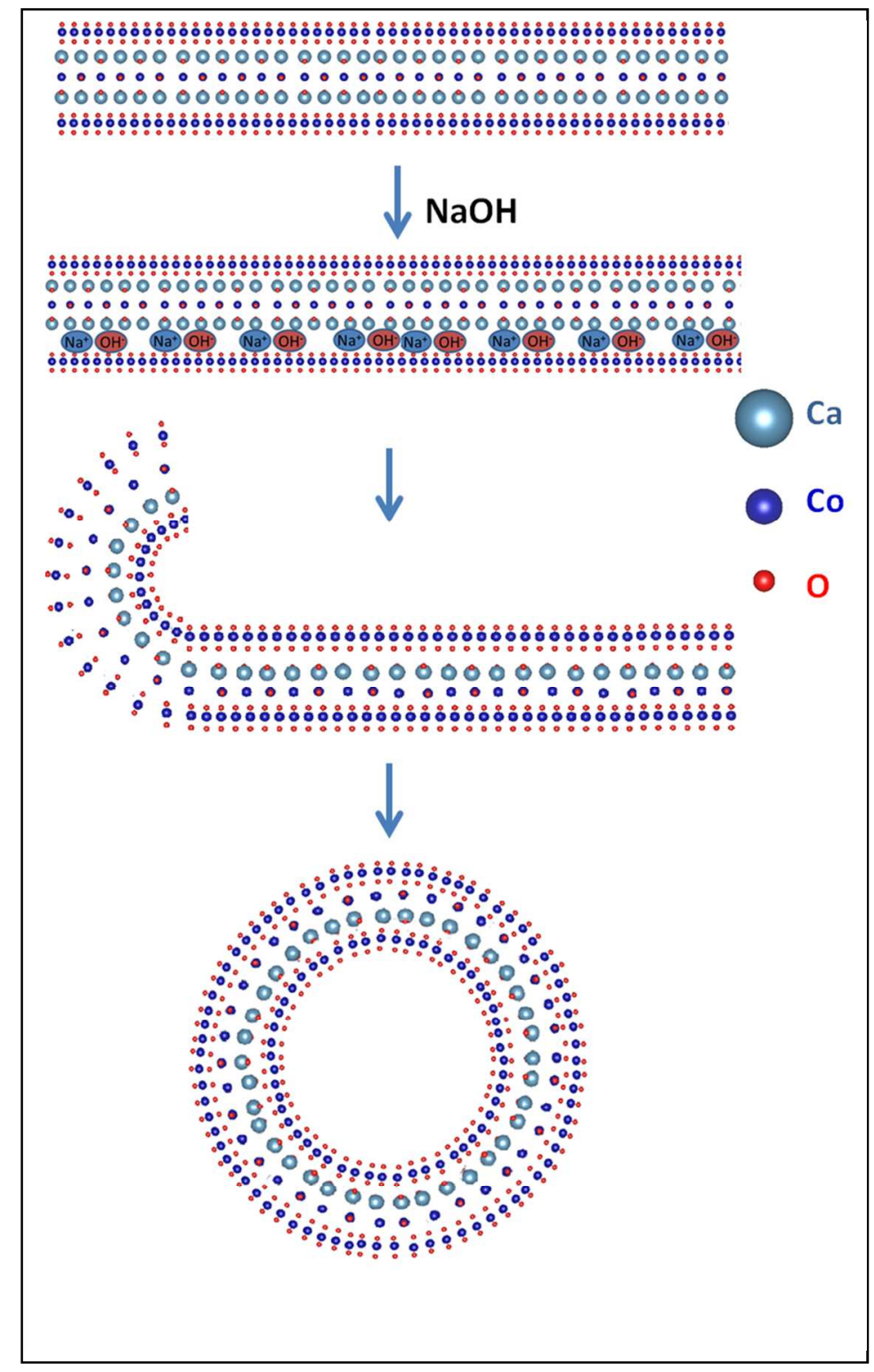

Figure 7. Schematic illustration for the formation mechanism of $\mathrm{CaCoO}_{2}-\mathrm{CoO}_{2}$ nanotubular structures. 


\section{CONCLUSIONS}

We synthesized and characterized a family of oxide nanotubes from misfit compounds leading to crystal phases with hitherto unknown composition. The compositional and structural details of these nanotubes were elucidated using a combination of several electron microscopy techniques and DFT calculations. The excellent agreement between DFT calculations, high-resolution microscopy, and spatially-resolved EELS spectroscopy allowed us to clearly establish that the nanotubes consist of alternate stacking of layers of hexagonal $\mathrm{CoO}_{2}$ and $\mathrm{CaCoO}_{2}$ along the $c$ axis. Furthermore, the $\mathrm{CaCoO}_{2}$ layer displays a distinctly anisotropic structure that may be viewed as quasi-1D, puckered chains along the $a$-axis. DFT calculations further predict that the obtained MLC phase is semiconducting in nature in contrast with the bulk phase which is metallic. Through systematic synthetic studies, we have proposed a formation mechanism for these cobalt oxide-based nanotubes. The driving forces for rolling of the layered structure arise from chemically-induced asymmetry in the structure along with misfit stresses between the subsystems. This formation mechanism is likely applicable to other MLCs and could be exploited broadly in realizing other oxide-based misfit nanotubes in the future. The synthesized oxide nanotubes are of potential interest for applications in the field of thermoelectrics and optoelectronics, and more generally for fundamental studies of physics of low-dimensional oxides.

\section{EXPERIMENTAL SECTION}

Synthesis. The synthesis of samples containing $\mathrm{CaCoO}_{2}-\mathrm{CoO}_{2}$ was performed in three steps. Cobalt acetate was first dissolved in Milli Q water with calcium acetate in 4:3 ratios. This 
solution was heated to $90{ }^{\circ} \mathrm{C}$ under stirring until a gel was formed. The gel was subsequently subjected to heat treatment at $900{ }^{\circ} \mathrm{C}$ for $20 \mathrm{~h}$ to produce $\mathrm{Ca}_{3} \mathrm{Co}_{4} \mathrm{O}_{9}\left(\mathrm{Ca}_{2} \mathrm{CoO}_{3}-\mathrm{CoO}_{2}\right)$ powders as confirmed by the XRD pattern (see Fig. S1 in the Supporting Information - SI). Thereafter, the powder was subjected to hydrothermal treatment under basic conditions to obtain nanotubes. In a typical reaction, $100 \mathrm{mg}$ of $\mathrm{Ca}_{3} \mathrm{Co}_{4} \mathrm{O}_{9}$ precursor was sonicated in $17 \mathrm{ml} \mathrm{NaOH}$ solution (700 mg) for $10 \mathrm{~min}$. This suspension was transferred to a Teflon lined autoclave, heated at $200{ }^{\circ} \mathrm{C}$ for 12 $\mathrm{h}$, and then allowed to cool naturally to room-temperature. The product was washed with Milli Q water several times and centrifuged to bring down to $\mathrm{pH} 7$.

Characterization. Powder X-Ray diffraction (XRD) analysis of the product was performed with a Rigaku diffractometer (TTRAX III). The nanotubes were imaged by Zeiss scanning electron microscope (SEM) model Ultra V55. Preliminary conventional transmission electron microscopy (TEM) analysis was done with Philips CM120 operating at $120 \mathrm{kV}$. High resolution TEM (HRTEM) and selected area electron diffraction (SAED) analyses were done using an FEI Tecnai F30-UT operated at $300 \mathrm{kV}$ and by FEI Tecnai F20 operating at $200 \mathrm{kV}$, equipped with high angle annular dark field (HAADF) detector for scanning TEM (STEM).High-resolution STEM imaging (HR-STEM) and spatially-resolved electron energy-loss spectroscopy (SREELS) were performed using an FEI Titan Low-Base microscope operated at $200 \mathrm{kV}$ and equipped with a $C_{s}$ probe corrector, a monochromator, an ultra-bright X-FEG electron source, and a Gatan Tridiem ESR 866 EELS spectrometer. HR-STEM imaging was performed by using HAADF and annular bright field $(\mathrm{ABF})$ detectors. The inner and outer angles for HAADF imaging were 110 and 200 mrad, respectively. During SR-EELS analyses, the energy resolution was about $1 \mathrm{eV}$ with a dispersion of $0.5 \mathrm{eV} /$ pixel. Spectra were collected in STEM mode, using spectrum image and line scan modes. ${ }^{24}$ For most of the EELS experiments, the convergence and 
collection angles were 18 and $10 \mathrm{mrad}$, respectively. The EELS datasets were then denoised with the open-source program Hyperspy by using principal component analysis routines. ${ }^{25,26}$ HAADF image simulation was performed by using the QSTEM software ${ }^{27}$ with the experimental settings of the Titan Low Base as inputs. For this purpose, the thermal diffuse scattering parameter was fixed at 30 for a temperature of $300 \mathrm{~K}$.

Computational. Density functional theory (DFT) calculations were performed using the Vienna Ab Initio Simulation Package (VASP). ${ }^{28,29}$ The projector-augmented wave method ${ }^{30,31}$ was used to represent the nuclei plus core electrons, the valence configurations for $\mathrm{Ca}$, $\mathrm{Co}$ and $\mathrm{O}$ being $3 s^{2} 3 p^{6} 4 s^{2}, 3 d^{8} 4 s^{1}$, and $2 s^{2} 2 p^{4}$, respectively. From convergence studies, a kinetic energy cutoff of $525 \mathrm{eV}$ was selected with a $\Gamma$-centered $6 \times 6 \times 3 k$-pointmesh for Brillouin zone sampling. A Gaussian smearing of $0.05 \mathrm{eV}$ was used to accelerate electronic convergence, and electronic wave functions were converged to $10^{-4} \mathrm{eV}$. Electron exchange and correlation were described using the Perdew-Burke-Ernzerhof (PBE) form of the generalized-gradient approximation. ${ }^{32}$ To improve the accuracy of calculation for strongly correlated $d$-electrons of Co, we employed the rotationally-invariant $\mathrm{DFT}+\mathrm{U}($ here $\mathrm{PBE}+\mathrm{U})$ approach of Dudarev et al. ${ }^{33}$ with values of $U=5 \mathrm{eV}$ and $J=1 \mathrm{eV}$, which have been successfully employed before for calcium cobaltite $(\mathrm{CCO}) .{ }^{18,34}$ These onsite corrections were applied only to the Co $d$-electrons. Atomic positions and cell volume were relaxed with a force tolerance of $0.01 \mathrm{eV} / \AA$. Subsequent to structural relaxation, electronic wave functions were recalculated using a denser $\Gamma$-centered $13 \times 13 \times 6 k$-point mesh and the Blöchl tetrahedron method ${ }^{35}(0.05 \mathrm{eV}$ smearing) was employed for calculating the density of states. Charge distributions were examined using the Bader analysis procedure. ${ }^{36,37}$ To ensure that our structure was not trapped in spurious local minima-a well-known issue when dealing with spin-polarized systems-we initialized our calculations with multiple low- and 
high-spin states to converge upon the most likely magnetic ground state of the system. It is well known that DFT+U calculations can often get stuck in different local minima with various antiferromagnetic or ferrimagnetic configurations, and various spin states for transition metal atoms. $^{38,39}$ To minimize the possibility of our structures becoming trapped in spurious local minima, we initialized our calculations with multiple low- and high-spin states to converge upon the most likely magnetic ground state of the system.

\section{Supporting Information:}

Powder X-Ray diffraction (XRD) analysis of as synthesized $\mathrm{Ca}_{3} \mathrm{Co}_{4} \mathrm{O}_{9}$ materials.

SEM and (HR-)TEM images of $\left(\mathrm{CaCoO}_{2}\right)-\left(\mathrm{CoO}_{2}\right)$ nanotubes as-synthesized and annealed at $200^{\circ} \mathrm{C}$ are provided. STEM-ADF images and extra SR-EELS data recorded in one of these NTs.

TEM images of partially rolled $\mathrm{CaCoO}_{2}-\mathrm{CoO}_{2}$ nanotubes synthesized in hydrothermal conditions at $200{ }^{\circ} \mathrm{C}$ for $4 \mathrm{~h}$ are given.

TEM images of $\mathrm{NaOH}$ intercalated $\mathrm{Ca}_{3} \mathrm{Co}_{4} \mathrm{O}_{9}$ nanotubular structures accomplished by sonication in the presence of $\mathrm{NaOH}$.

Concerning the DFT calculations we include: (1) The atomic positions and lattice vectors for the relaxed $\left(\mathrm{CaCoO}_{2}\right)-\left(\mathrm{CoO}_{2}\right)_{2}$ and $\left(\mathrm{Ca}_{2} \mathrm{CoO}_{3}\right)_{2}-\left(\mathrm{CoO}_{2}\right)_{3}$ supercell structures, respectively. The number of valence electrons and the magnetization for each atom in these structures are also included and they are calculated by integration over Bader volumes. (2) The spin-resolved total density of states projected over the d-orbitals of these $\mathrm{Co}$ atoms for $\left(\mathrm{CaCoO}_{2}\right)-\left(\mathrm{CoO}_{2}\right)_{2}$ supercell, and for the individual $\mathrm{CaCoO}_{2}$ and $\mathrm{CoO}_{2}$ layers. 
"This material is available free of charge via the Internet at http://pubs.acs.org."

\title{
AUTHOR INFORMATION
}

\section{Corresponding Authors}

*Email: arenal@unizar.es ; reshef.tenne@weizmann.ac.il ; ashwin@engin.umass.edu

\begin{abstract}
Author Contributions
The manuscript was written through contributions of all authors. All authors have given approval to the final version of the manuscript. $\ddagger$ These authors contributed equally.
\end{abstract}

Acknowledgments: We thank Dr. Y. Feldman for the X-ray analysis, Dr. R. Popovitz-Biro for help in HRTEM measurements. The Israel Science Foundation (grant 265/12); the H. Perlman Foundation; the Irving and Azelle Waltcher Foundations in honor of Prof. M. Levy and the Irving and Cherna Moskowitz Center for Nano and Bio-Nano Imaging. L.S.P. would like to thank the PBC Program of the 469 Government of Israel and the Dean of the chemistry faculty, 470 Weizmann Institute of Science, for a postdoctoral fellowship. The STEM and EELS studies were conducted at the Laboratorio de Microscopias Avanzadas, Instituto de Nanociencia de Aragon, Universidad de Zaragoza, Spain. Some of the research leading to these results has received funding from the European Union Seventh Framework Programme under Grant Agreement 312483- ESTEEM2 (Integrated Infrastructure Initiative - I3) and from the European Union H2020 Grant Agreement 696656 Graphene Flagship. R. A. gratefully acknowledges the support from the Spanish Ministerio de Economia y Competitividad (FIS2013-46159-C3-3-P) and from the European Union H2020 program ETN project "Enabling Excellence" Grant 
Agreement 642742. AR gratefully acknowledges supercomputing resources through the

Massachusetts Green High Performance Computing Center and useful discussions with Kevin Kittilstved.

\section{References}

(1) Rao, C. N. R.; Raveau, B.: Transition Metal Oxides. Second Edition ed.; Wiley-VCH, 1999.

(2) Motohashi, T.; Ueda, R.; Naujalis, E.; Tojo, T.; Terasaki, I.; Atake, T.; Karppinen, M.; Yamauchi, H. Unconventional Magnetic Transition and Transport Behavior in $\mathrm{Na}_{0.75} \mathrm{CoO}_{2}$. Phys. Rev. B 2003, 67, 064407.

(3) Nitta, N.; Wu, F.; Lee, J. T.; Yushin, G. Li-ion Battery Materials: Present and Future. Mater. Today 2015, 18, 252-264.

(4) Sugiura, K.; Ohta, H.; Nomura, K.; Hirano, M.; Hosono, H.; Koumoto, K. High Electrical Conductivity of Layered Cobalt Oxide $\mathrm{Ca}_{3} \mathrm{Co}_{4} \mathrm{O}_{9}$ Epitaxial Films Grown by Topotactic Ion-Exchange Method. Appl. Phys. Lett. 2006, 89, 032111.

(5) Terasaki, I.; Sasago, Y.; Uchinokura, K. Large Thermoelectric Power in $\mathrm{NaCO}_{2} \mathrm{O}_{4}$ Single Crystals. Phys. Rev. B 1997, 56, R12685.

(6) Masset, A. C.; Michel, C.; Maignan, A.; Hervieu, M.; Toulemonde, O.; Studer, F.; Raveau, B.; Hejtmanek, J. Misfit-Layered Cobaltite with an Anisotropic Giant Magnetoresistance: $\mathrm{Ca}_{3} \mathrm{Co}_{4} \mathrm{O}_{9}$. Phys. Rev. B 2000, 62, 166.

(7) Takada, K.; Sakurai, H.; Takayama-Muromachi, E.; Izumi, F.; Dilanian, R. A.; Sasaki, T. Superconductivity in Two-Dimensional $\mathrm{CoO}_{2}$ Layers. Nature 2003, 422, 53-55.

(8) Liu, C.-J.; Huang, L.-C.; Wang, J.-S. Improvement of the Thermoelectric Characteristics of Fe-Doped Misfit-Layered $\mathrm{Ca}_{3} \mathrm{Co}_{4-\mathrm{x}} \mathrm{Fe}_{\mathrm{x}} \mathrm{O}_{9+\delta}(\mathrm{x}=0,0.05,0.1$, and 0.2). Appl. Phys. Lett. 2006, 89, 204102.

(9) Shikano, M.; Funahashi, R. Electrical and Thermal Properties of Single-Crystalline $\left(\mathrm{Ca}_{2} \mathrm{CoO}_{3}\right)_{0.7} \mathrm{CoO}_{2}$ with a $\mathrm{Ca}_{3} \mathrm{Co}_{4} \mathrm{O}_{9}$ Structure. Appl. Phys. Lett. 2003, 82, 1851-1853.

(10) Schrade, M.; Fjeld, H.; Finstad, T. G.; Norby, T. Electronic Transport Properties of $\left[\mathrm{Ca}_{2} \mathrm{CoO}_{3-\delta}\right]_{\mathrm{q}}\left[\mathrm{CoO}_{2}\right]$. J. Phys. Chem. C 2014, 118, 2908-2918.

(11) Hicks, L. D.; Dresselhaus, M. S. Thermoelectric Figure of Merit of a OneDimensional Conductor. Phys. Rev. B 1993, 47, 16631-16634.

(12) Panchakarla, L. S.; Radovsky, G.; Houben, L.; Popovitz-Biro, R.; Dunin-Borkowski, R. E.; Tenne, R. Nanotubes from Misfit Layered Compounds: A new family of Materials with Low Dimensionality. J. Phys. Chem. Lett. 2014, 5, 3724-3736.

(13) Radovsky, G.; Popovitz-Biro, R.; Staiger, M.; Gartsman, K.; Thomsen, C.; Lorenz, T.; Seifert, G.; Tenne, R. Synthesis of Copious Amounts of $\mathrm{SnS}_{2}$ and $\mathrm{SnS}_{2} / \mathrm{SnS}$ Nanotubes with Ordered Superstructures. Angew. Chem., Int. Ed. 2011, 50, 12316-12320. 
(14) Panchakarla, L. S.; Popovitz-Biro, R.; Houben, L.; Dunin-Borkowski, R. E.; Tenne, R. Lanthanide-Based Functional Misfit-Layered Nanotubes. Angew. Chem., Int. Ed. 2014, $536920-6924$.

(15) Ewels, P.; Sikora, T.; Serin, V.; Ewels, C. P.; Lajaunie, L. A Complete Overhaul of the EELS and XAS Database: eelsdb.eu. Microsc. Microanal. 2016, In Press, DOI: $10.1017 / \mathrm{S} 1431927616000179$

(16) Lajaunie, L.; Boucher, F.; Dessapt, R.; Moreau, P. Quantitative Use of Electron Energy-Loss Spectroscopy Mo-M $\mathrm{M}_{2,3}$ Edges for the Study of Molybdenum Oxides. Ultramicroscopy 2015, 149, 1-8.

(17) Panchakarla, L. S.; Lajaunie, L.; Tenne, R.; Arenal, R. Atomic Structural Studies on Thin Single-Crystalline Misfit-Layered Nanotubes of $\mathrm{TbS}_{-} \mathrm{CrS}_{2}$. J. Phys. Chem. C 2015, In Press, DOI:10.1021/acs.jpcc.5b05811

(18) Rébola, A.; Klie, R.; Zapol, P.; Öğüt, S. First-Principles Study of the Atomic and Electronic Structures of Misfit-Layered Calcium Cobaltite $\left(\mathrm{Ca}_{2} \mathrm{CoO}_{3}\right)\left(\mathrm{CoO}_{2}\right)_{1.62}$ Using Rational Approximants. Phys. Rev. B 2012, 85, 155132.

(19) Asahi, R.; Sugiyama, J.; Tani, T. Electronic Structure of Misfit-Layered Calcium Cobaltite. Phys. Rev. B 2002, 66, 155103.

(20) Pauling, L. The Structure of the Chlorites. Proc. Natl. Acad. Sci. U.S.A. 1930, 16, 578582.

(21) Bates, T. F.; Sand, L. B.; Mink, J. F. Tubular Crystals of Chrysotile Asbestos. Science 1950, 111, 512-513.

(22) Zhang, S.; Peng, L. M.; Chen, Q.; Du, G. H.; Dawson, G.; Zhou, W. Z. Formation Mechanism of $\mathrm{H}_{2} \mathrm{Ti}_{3} \mathrm{O}_{7}$ Nanotubes. Phys. Rev. Lett. 2003, 91, 256103.

(23) Du, G.; Chen, Q.; Yu, Y.; Zhang, S.; Zhou, W.; Peng, L.-M. Synthesis, Modification and Characterization of $\mathrm{K}_{4} \mathrm{Nb}_{6} \mathrm{O}_{17}$-Type Nnanotubes. J. Mater. Chem. 2004, 14, 14371442

(24) Jeanguillaume, C.; Colliex, C. Spectrum-image: The Next Step in EELS Digital Acquisition and Processing. Ultramicroscopy 1989, 28, 252-257.

(25) Peña, F. d. 1.; Burdet, P.; Ostasevicius, T.; Sarahan, M.; Nprd, M.; Taillon, V. T. F. J.; Eljarrat, A.; Mazzucco, S.; Donvail, G.; Zagonel, L. F.; Walls, M.; Iyengar, I.: HyperSpy: Multidimensional Data Analysis Toolbox. 2015.

(26) Arenal, R.; de la Peña, F.; Stéphan, O.; Walls, M.; Tencé, M.; Loiseau, A.; Colliex, C. Extending the Analysis of EELS Spectrum-Imaging Data, from Elemental to Bond Mapping in Complex Nanostructures. Ultramicroscopy 2008, 109, 32-38.

(27) Koch, C. T. Determination of Core Structure Periodicity and Point Defect Density Along Dislocations. Arizona State University, 2002.

(28) Kresse, G.; Furthmüller, J. Efficiency of ab-initio Total Energy Calculations for Metals and Semiconductors using a Plane-Wave Basis Set. Comp. Mater. Sci. 1996, 6, $15-50$.

(29) Kresse, G.; Furthmüller, J. Efficient Iterative Schemes for ab-initio Total-Energy Calculations using a Plane-Wave Basis Set. Phys. Rev. B 1996, 54, 11169-11186.

(30) Blöchl, P. E. Projector Augmented-Wave Method. Phys. Rev. B 1994, 50, 1795317979.

(31) Kresse, G.; Joubert, D. From Ultrasoft Pseudopotentials to the Projector AugmentedWave Method. Phys. Rev. B 1999, 59, 1758-1775. 
(32) Perdew, J. P.; Burke, K.; Ernzerhof, M. Generalized Gradient Approximation Made Simple. Phys. Rev. Lett. 1996, 77, 3865-3868.

(33) Dudarev, S. L.; Botton, G. A.; Savrasov, S. Y.; Humphreys, C. J.; Sutton, A. P. Electron-Energy-Loss Spectra and the Structural Stability of Nickel Oxide: An LSDA+U Study. Phys. Rev. B 1998, 57, 1505-1509.

(34) Rébola, A.; Klie, R. F.; Zapol, P.; Ögüut, S. Phonon and Thermal Transport Properties of the Misfit-Layered Oxide Thermoelectric $\mathrm{Ca}_{3} \mathrm{Co}_{4} \mathrm{O}_{9}$ from First Principles. Appl. Phys. Lett. 2014, 104, 251910.

(35) Blöchl, P. E.; Jepsen, O.; Andersen, O. K. Improved Tetrahedron Method for Brillouin-Zone Integrations. Phys. Rev. B 1994, 49, 16223-16233.

(36) Bader, R. F. W. A Quantum Theory of Molecular Structure and its Applications. Chem. Rev. 1991, 91, 893-928.

(37) Henkelman, G.; Arnaldsson, A.; Jónsson, H. A Fast and Robust Algorithm for Bader Decomposition of Charge Density. Comp. Mater. Sci. 2006, 36, 354-360.

(38) Ylvisaker, E. R.; Pickett, W. E.; Koepernik, K. Anisotropy and Magnetism in the LSDA+U Method. Phys. Rev. B 2009, 79, 035103.

(39) Meredig, B.; Thompson, A.; Hansen, H. A.; Wolverton, C.; van de Walle, A. Method for Locating Low-Energy Solutions within DFT+U. Phys. Rev. B 2010, 82, 195128. 

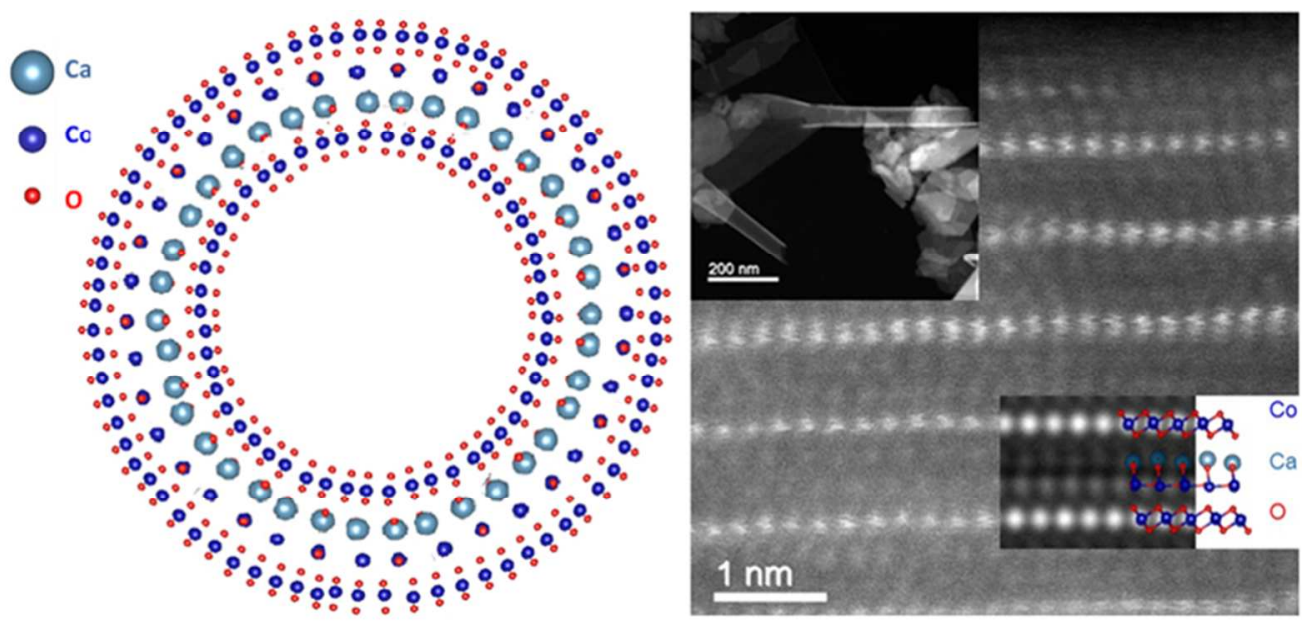

ToC graphic

$325 \times 152 \mathrm{~mm}(68 \times 68$ DPI $)$ 